\section{Synthese des Meteloidins}

Von Karl Zeile und Alex Heusner

Wissenschaftliche Abteilung der Firma C. H. Boehringer Sohn, Ingelheim a. Rh.

(Z. Naturforschg. 12 b, 661 [1957]; eingegangen am 31. Mai 1957)

Die Totalsynthese der Tropan-Alkaloide erfordert als letzte Phase eine Veresterung des Alkamins mit einer aliphatischen oder aromatischen Säure.

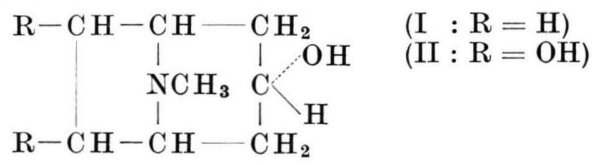

Bei Tropin (I) selber bereitet diese Veresterung keine prinzipiellen Schwierigkeiten, so daß alle davon abgeleiteten Alkaloide auch vollsynthetisch bereitet werden konnten. Dagegen ist das im Tropan-Anteil zweifach hydroxylierte Alkaloid Meteloidin (6.7-Dihydroxytropin-3-tiglinsäureester; VIII) bis heute noch nicht synthetisiert worden, obwohl sein Alkamin Teloidin (6.7-Dihydroxy-tropin; II) schon seit einer Reihe von Jahren synthetisch zugänglich ist ${ }^{1}$. Offensichtlich hat ein Mangel an rationellen Methoden zum intermediären Schutz der Hydroxylgruppen des Pyrrolidinringes diese Synthese bisher verhindert ${ }^{2}$.

Wir haben nunmehr gefunden, daß sich in der Tropan-Reihe benachbarte cis-ständige Hydroxylgruppen in Form ihres cyclischen Carbonats schützen lassen. Derartige Cyclocarbonate werden in einfacher Weise durch Erhitzen ihrer Hydrochloride in wäßriger Lösung wieder verseift, ohne daß dabei andere Esterbindungen angegriffen werden. Unter Anwendung dieser Methodik gelang es uns, auf folgendem Wege erstmalig Meteloidin (VIII) darzustellen ${ }^{3}$ :<smiles>CN1CC2CC1C1OC(OC3CC4CCC3C4O)OC2C1O</smiles>
(III)

1 C.. Schöpf u. W. Arnold, Liebigs Ann. Chem. 558, 109 [1947].

2 Das vom einfach hydroxylierten 6-Hydroxy-tropin abgeleitete Alkaloid Valeroidin ist dagegen kürzlich von G. Fodor, J. Tóth, I. Koczor u. I. Vincze (Chem. and Ind. 1955, 1260 ; J. chem. Soc. [London] 1957, 1349) synthetisch erhalten worden; die isolierte Hydroxylgruppe des Pyrrolidinrings ist hier als Phenylurethan reversibel geschützt worden.

3 J. C. Sheehan u. E. R. Bissell (J. organ. Chemistry 19, 270 [1954]) haben gezeigt, daß sich die benachbarten Hydroxylgruppen des Teloidins (II) auch als Benzyliden-Verbindung reversibel schützen lassen. Da die Benzylidengruppe jedoch nach erfolgter Veresterung an C-3 hydrogenolytisch entfernt werden muß, konnten die amerikanischen Autoren auf diesem Weg naturgemäß nicht Meteloidin (VIII) selber, sondern nur dessen Dihydroderivat (VIII, mit dem Rest der $\alpha$-Methylbuttersäure an Stelle des

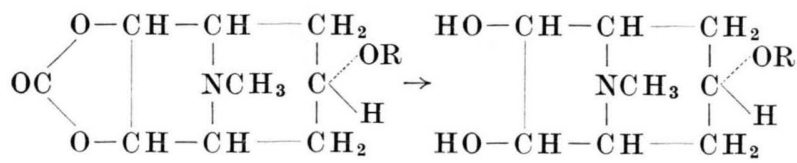

$$
\begin{aligned}
& (\mathrm{V}: \mathrm{R}=-\mathrm{H}) \\
& \left(\mathrm{VI}: \mathrm{R}=-\mathrm{COC}\left(\mathrm{CH}_{3}\right)=\mathrm{CH} \cdot \mathrm{CH}_{3}\right) \\
& \left(\mathrm{VII}: \mathrm{R}=-\mathrm{COCH}_{3}\right) \\
& \left(\mathrm{VIII}: \mathrm{R}=-\mathrm{COC}\left(\mathrm{CH}_{3}\right)=\mathrm{CH} \cdot \mathrm{CH}_{3}\right) \\
& \left(\mathrm{IX}: \mathrm{R}=-\mathrm{COCH}_{3}\right)
\end{aligned}
$$

Teloidin (III) ${ }^{\mathbf{1}, 4}$ wurde durch natriumkatalysierte Umesterung mit Diäthylcarbonat in sein Cyclocarbonat (IV) verwandelt (aus Chloroform/Petroläther farblose Blöcke vom Schmp. $218-219^{\circ}$ ), das sich in äthanolischer Lösung mit Raney-Nickel unter Druck zu Teloidin-carbonat (V) hydrieren ließ (aus Aceton/ Petroläther farblose Nadeln vom Schmp. $198-200^{\circ}$ ). Umsetzung von (V) mit Tiglinsäureanhydrid in siedendem Toluol unter Zusatz von Pyridin führte zu Meteloidin-carbonat (VI) (aus Benzol/Petroläther farblose gefiederte Nadeln vom Schmp. $150-151^{\circ}$ ), und dessen Hydrochlorid (aus Methanol/Isopropyläther farblose Blättchen vom Schmp. 241, $5^{\circ}$ ) ergab beim Kochen seiner wäßrigen Lösung Meteloidin (VIII). Die Identifizierung mit dem natürlichen Produkt geschah durch Analyse, Misch-Schmp., Papierchromatogramm und URSpektrum.

In analoger Weise wurde (V) noch in 3-Acetylteloidin-6.7-cyclocarbonat (VII) umgewandelt (Schmp. $\left.148-150^{\circ}\right)$, dessen Hydrochlorid, Schmp. 266 ${ }^{\circ}$, sich zu Teloidin-3-acetat (IX), Schmp. $161-163^{\circ}{ }^{5}$, partiell verseifen ließ.

Das noch unbekannte ${ }^{6}$ Anhydrid der Tiglinsäure, Sdp. $0,790-95^{\circ}$, wurde durch Umsetzung von Tiglylchlorid $^{7}$ mit Acetanhydrid nach dem Verfahren von Sonntag und Mitarbb. ${ }^{8}$ gewonnen.

Eine ausführliche Mitteilung über im Pyrrolidinring substituierte Tropanester ist in Vorbereitung.

Tiglinsäurerestes) gewinnen. Vgl. auch A. Heusner, Chem. Ber. 87, 1032 [1954].

4 J. C. Sheehan u. B. M. Bloom, J. Amer. chem. Soc. 74, 3825 [1952].

5 Schmp. der kristallbenzolhaltigen Verbindung. Das lösungsmittelfreie Produkt schmilzt nach Sheehan u. Bissell ${ }^{3}$ bei $178,5-179,5^{\circ}$.

6 Bisher war lediglich das Anhydrid der Angelicasäure beschrieben worden: L. Chrozza, Liebigs Ann. Chem. 86, 259 [1853] ; vgl. auch R. E. Buckies, G. V. Mock u. L. Locatelli JR., Chem. Reviews 55, 659 [1955].

7 G. Barger. Wm. F. Martin u. Wm. Mitchell, J. chem. Soc. [London] 1937, 1820.

8 N. O. Sonntag, J. R. Trowbridge u. I. J. Krems, J. Amer. Oil Chemists' Soc. 31, 151 [1954]; vgl. auch F. Zetzsche, F. Enderlin, C. Fü̈tsch u. E. Menzi, Helv. chim. Acta 9, 181 [1926]. 